\title{
The gas-solid Joule-Thomson Effect
}

\author{
Robert A. Plerotti and Thomas R. Rybolt \\ School of Chemistry, Georgla Institute of Technology, Atlanta, G A 30332 \\ Department of Chemistry, The University of Tennessee, Chattanooga, TN 37402
}

\begin{abstract}
The magnitude of interaction of a gas with a solid is expected to cause an aerosol created by dispersing a fine powder in a gas to have unique thermodynamic properties not found in pure or mixed gases. The virial equation of state associated with a gas-solid aerosol or dusty gas is obtained from statistical thermodynamic considerations. The theoretical model of the aerosol is that of a two component fluid made up of solid particles and gas molecules. The aerosol virial equation of state is used to derive an expression for the Joule-Thomson effect associated with a gas-solid dispersion. The magnitude of the gas-solid Joule-Thomson effect is expressed in terms of gas-gas and gas-solid virial coefficients. Previous adsorption data for an argon-porous carbon system is used to obtain gas-solid virial coefficients and to predict the magnitude of the gas-solid Joule-Thomson effect. A significant enhancement of the Joule-Thomson effect is predicted for gas-solid systems which display strong interactions. An apparatus was constructed to disperse a fine powder in a flowing gas and measure the thermal changes associated with a pressure drop across a glass orifice. The gas-solid Joule-Thomson effect was examined for 12 different gas-sol1d systems at a temperature of $302 \mathrm{~K}$. Measurements for a serles of aerosols composed of four different carbon adsorbents with argon, nitrogen and carbon dioxide confirm the effect and pose new questions.
\end{abstract}

\section{INTRODUCTION}

Over the years many theoretical and experimental studies of the JouleThomson effect have been carried out on a wide variety of pure and mixed gases. There has been no theoretical or experimental study of the JouleThomson effect associated with a gas-solid dispersion. In this paper a statistical thermodynamic theory of the Joule-Thomson effect for a two component fluid containing solid particles and gas molecules is presented. The Joule-Thomson coefficlent associated with a gas-solid dispersion (aerosol) is expressed in terms of gas-gas and gas-solid virial coefficlents, the heat capacity of the gas-solld mixture and the concentration of the solid component. A significant enhancement of the Joule-Thomson effect is predicted for those systems which display a favorable gas-solid interaction.

\section{THEORY}

General Conditions. In order to develop an expression for the Joule-Thomson coefficlent of a gas-solid dispersion it is first useful to derive a virial equation of state for an aerosol (ref. 1). Statistical thermodynamic modeling of a gas-solid dispersion provides the best means of developing a proper virial equation of state. A correct statistical treatment will of necessity include the gas-gas interactions as manifest in deviations from gas ideality and the gas-solid interactions as manifest in physical adsorption.

Ut1lizing the ensemble method of Glbbs, a virial equation of state for a solid powder dispersed in a gas is derived. The treatment presented uses the grand partition function and is based on the following assumptions: 
(a) The system of interest is a two component fluld phase composed of a molecular gaseous component and a particulate component. The solid particles are formally treated as giant molecules.

(b) The molecular component is an imperfect gas capable of interacting with the particulate component and 1tself through dispersion forces.

(c) The particulate component is dilute enough to exclude particleparticle interaction.

The grand partition function for a gas-solid mixture may be considered analogous to the grand partition function of a two component imperfect gas where the gaseous molecules of the second component are replaced by solid particles. The appropriate grand partition function for the model above in terms of canonical partition functions is glven by (ref. 2)

$$
\Xi\left(\lambda_{\mathrm{g}}, \lambda_{\mathrm{s}}, \mathrm{V}, \mathrm{T}\right)=\sum_{\mathrm{N}_{\mathrm{s}}>0} \quad \mathrm{~N}_{\mathrm{g}} \geqslant 0{ }_{\mathrm{N}}, \mathrm{N}_{2 \mathrm{~s}}(\mathrm{~V}, \mathrm{~T}) \lambda_{\mathrm{g}}{ }^{\mathrm{Ng}} \lambda_{\mathrm{s}}{ }^{\mathrm{Ns}}
$$

where $\lambda_{\mathrm{g}}$ is the absolute activity of the gas, $\lambda_{\mathrm{s}}$ is the absolute activity of the particulate sol1d, $\mathrm{N}_{\mathrm{g}}$ is the number of gas molecules, $\mathrm{N}_{\mathrm{s}}$ is the number of particles, and $Q_{\mathrm{Ns}}, \mathrm{Ng}$ is the canonical partition $\mathrm{N}_{\mathrm{s}}, \mathrm{N}_{\mathrm{g}}$ for $\mathrm{N}_{\mathrm{s}}$ solid particles and $\mathrm{N}_{\mathrm{g}}$ gas molecules. As in the conventional statistical treatment of fluids, it proves convenient to replace the canonical partition functions with the appropriate configuration integrals. In addition, two new activities, $z_{s}$ activity of the particulate solid, and $z_{g}$ activity of the gas, are introduced. These activities become equivalent to their respective number densities $\rho_{s}$ number density of solid particles, and $\rho_{g}$ number density of gas molecules, in the limit as the densities go to zero.

The statistical thermodynamic treatment developed is applicable to any two component fluids. This generalization is lost by assuming the particulate component is very dilute. If the particulate component is dilute enough to exclude particle-particle interaction then any system within the grand ensemble contains e1ther no particle or one particle. Thus, 1 is allowed only values of zero or one. As in the treatment of an imperfect gas, there is no restriction on the number of gas molecules within any system of the ensemble. Following established procedures and utilizing the characteristic function for the grand ensemble, $P V / R T=1$ I $\Xi$ and replacing $\Xi$, one may expand the logarithm, divide by $V$, and group like powers of the $z$ activities and obtain

$$
\mathrm{P} / \mathrm{kT}=\mathrm{z}_{\mathrm{s}}+\sum_{j>1}\left(\mathrm{~b}_{1 j} \mathrm{z}_{\mathrm{s}}+\mathrm{b}_{0 j}\right) \mathrm{z}_{\mathrm{s}}^{j}
$$

where $b_{1 j}$ is a cluster integral, 1 is the number of solid particles, and $f$ is the number of gas molecules. The cluster integrals are related to the configuration integrals for mixtures in the usual manner (ref 2 ).

Virial Equation for an Aerosol. To obtain a useful equation of state it is necessary to express the preceding equation as a power series in the number density of the gas, $\rho_{g}$ and the number density of solid particles, $\rho_{s}$ and eliminate the use of the $z$ activities. The number densities and activities are related by

$$
\rho_{1}=z_{1}\left[\partial(P / k T) / \partial z_{1}\right]\{z\}, V, T
$$

Utilizing equations (2) and (3) yields after mathematical manipulation

$$
P / k T=\rho_{s}+\sum_{j>1} C_{j}(T) \rho_{g}^{j}
$$

where the $\mathrm{C}_{\mathrm{g}}$ 's are collections of cluster integrals (ref. 1 ).

To develop a statistical theory of the gas-solid Joule-Thomson effect it. is necessary to consider the influence of both gas-gas and gas-solid 
interactions. Gas-solid interactions due to van der Wals forces give rise to physical adsorption. In physical adsorption the number of molecules adsorbed is defined as the excess number of molecules in a volume due to the interaction of gas molecules with the adsorbent (ref. 3). In the virial coefficient treatment of physical adsorption, the number of moles adsorbed per gram of adsorbent, $n_{a}$ is given by

$$
n_{a}=\sum_{1>2} B_{1 s}(f / R T)^{1-1}
$$

where $f$ is the fugacity, $R$ is the gas constant and $B_{1 s}$ is the (1)th gassolid virial coefficient (ref. 4). The interaction of a single gas molecule with a solid adsorbent is reflected in $\mathrm{B}_{2 \mathrm{~s}}$, the second gas-solid virial coefficient, while $B_{3 s}$, the third gas-solid virlal coefficient involves a pair of molecules interacting with the solid, $B_{4 s}$ involves a triplet of gas molecules, etc. It should be noted that the gas-solid virial coefficients are usually expressed in terms of per gram of solid.

Combining equations (6) and (7) and rearranging yields

$$
\mathrm{PV} / \mathrm{RT}=1+\left\{\left(\mathrm{B}_{2 \mathrm{~g}}-\mathrm{WB}_{2 \mathrm{~s}}\right)(\mathrm{P} / \mathrm{RT})\right\}+0\left\{\mathrm{P}^{2}\right\}
$$

where $O\left\{P^{2}\right\}$ are terms of order of $P^{2}$ or greater and $w$ is the mass of solid per mole of gas. Equation (8) is the virial equation of state for the gassolid dispersion (aerosol) (ref. 1).

Gas-Solid Joule-Thomson Coefficient. The virial equation of state for an aerosol can be used to obtain the gas-solid Joule-Thomson coefficlent since

$$
\mu_{g s} C_{P}=T(\partial V / \partial T)_{p}-V
$$

where $C_{P}$ is the constant pressure molar heat capacity and $\mu J T$ is the JouleThomson coefficient. Solving (8) for $V$ and substituting the result into (9) Gives

$$
\mu_{g s} C_{g s}=\left\{\left(\mathrm{TB}_{2 g^{\prime}}-\mathrm{B}_{2 g^{\prime}}\right)+w\left(\mathrm{~B}_{2 s}-\mathrm{TB}_{2 s^{\prime}}\right)\right\}+0\{\mathrm{P}\}
$$

where $\mathrm{C}_{\mathrm{gs}}$ is the constant pressure heat capacity for a gas-solid dispersion consisting of $w$ grams of particulate solid in one mole of gas and where $B_{2} s^{\prime}$ is the temperature derivative of $B_{2 s}$ (ref. 1). The first term in brackets represents the limiting value of the gas-solid Joule-Thomson coefficient at zero pressure.

Calculations of the Joule-Thomson Effect. At this point, one may ant1c1pate the possibility of greatly enhancing the Joule-Thomson effect of a gas by dispersing a fine powder into 1t. This effect is to be expected since the gas-solid interaction is typically much stronger than the gas-gas interaction. To explore this possibility specific numerical examples based on avallable data for an argon-powder systems are considered.

Cole and Everett (ref. 5) have reported experimental adsorption data for nitrogen and inert gases interacting with activated porous carbons. This data is in the temperature range of 275 to $400 \mathrm{~K}$ and pressure range up to 1 atm. Their reported Henry law constants as a function of temperature for the adsorption of argon by carbon C (a Saran carbon w1th a BET area of 1280 $\mathrm{m}^{2} / \mathrm{g}$ ) were used to obtain the values of $\mathrm{B}_{2 \mathrm{~s}}$ which were found to be described by the following equation

$$
\mathrm{B}_{2 \mathrm{~s}}=0.0521 \exp (-1692 / \mathrm{T})
$$

Calculated values of $B_{2 s}$ based on ( 9 ) agreed with the experimental values within 0.1 to $0.5 \%$. This equation was used to calculate $B_{2} s^{\prime}$. The values of ${ }^{\mathrm{JT}}$ calculated using equation (8) are shown in Fig. 1.

Data for adsorption of argon on a 5A-zeolite were determined by Yang and Plerott1 (ref. 6) over a wide temperature range and the procedure used in treating the Cole and Everett data were followed. Adsorption data for argon 
on P33 graphit1c carbon determined by Sams, Constabaris and Halsey were used to include a low surface area powder (ref. 7). Values of $\mu_{\mathrm{JT}}$ for these systems are shown in FIg. 1 .

There are several comments worth making concerning Fig. 1. All calculations are based upon $w$ equal to one gram of solid per liter of gas. The curve labelled Ar-Ar represents the Joule-Thomson coefficient for pure gaseous argon. The curve labelled Ar-P33 is the Joule-Thomson coefficient for argon interacting with a non-porous low surface area graphitic carbon and is based upon the data of Sams, Constabarls and Halsey. The curve labelled Ar-Porous $\mathrm{C}$ is based on the Cole and Everett data while the curve labelled Ar-5A is based upon the zeolite data of Yang and Plerotti. Clearly, the calculated effect indicates a major enhancement over the pure gas for the high surface area solids. The insert in FIg. I shows that for the non-porous graphite there is an attenuation in the Joule-Thomson effect at temperature above about $150 \mathrm{~K}$. The attenuation is primarliy a heat capacity effect. The substantial effect indicated that it would be worthwhile directly measuring gas-solid Joule-Thomson coefficients and accordingly an apparatus was designed and constructed to do this (ref. 8 ).

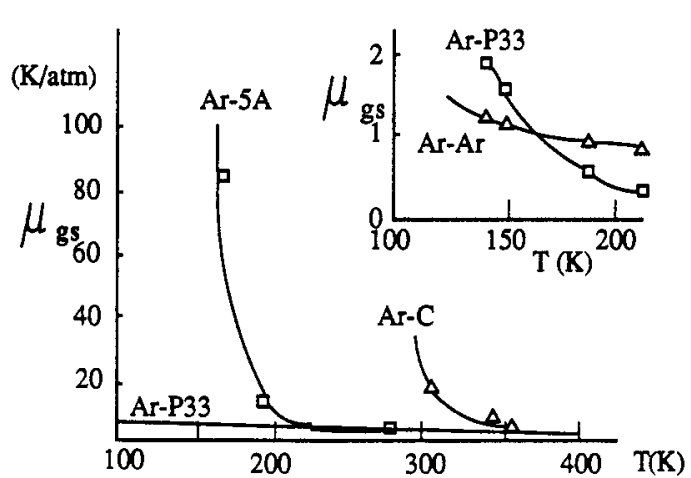

Fig. 1. Gas-solid Joule-Thomson Coefficients calculated for Argon interacting with charcoal (C), zeolite (5A) and graphite (P33).

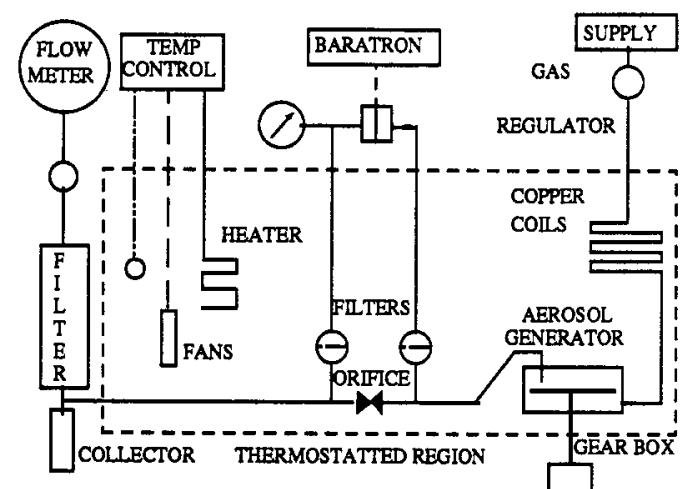

Fig. 2. Schematic diagram of apparatus for the measurentent of gas-solid Joule-Thomson coefficients.

\section{EXPERIMENTAL}

Apparatus. A schema of the gas-solid Joule-Thomson apparatus is shown in Fig. 2. With this apparatus it is possible to disperse a fine powder into a gas stream, flow the resultant mixture through an orifice, measure the absolute pressure and temperature of the flowing aerosol, measure the pressure drop and temperature change across the orifice, filter out the solid powder, and determine the flow rate of the gas. The portion of the apparatus which required temperature control was placed inside a thermostated box. The aerosol generator consisted of a metal chamber containing a rotating powder container, retaining partition, and pickup valve. Powder was entrained and deagglomerated by gas passing upward through the multiple small diameter passages in the pickup valve.

The flow system was designed for the gas-solid mixture to flow through a glass constriction. A fllter collected the particulate solid, and a wet test meter measured the flow rate of the gas. The gas passed through an inlet valve and was thermally equilibrated prior to entering the aerosol generator. The gas-solid mixture passed through a glass constriction tube ( $6 \mathrm{~mm}$ I.D. and $9.4 \mathrm{~mm} \mathrm{O.D.} \mathrm{with} \mathrm{a} 1 \mathrm{~mm} \mathrm{I.D.} \mathrm{orifice).} \mathrm{Branches} \mathrm{off} \mathrm{the}$ central tube were $10 \mathrm{~cm}$ before and after the constriction. Powder filters using medium porosity fritted glass disks were placed at each of these side tubes. The solid was separated from the gas by means of a filter and was weighed whlle the gas volume was measured with the wet test meter.

An MKS Baratron capacitance manometer was used to measure the pressure difference across the glass orifice. The high pressure and low pressure sldes of the capacitance head were connected to the upstream and downstream filters, respectively. A Bourdon Gauge was used to measure the pressure downstream of the orifice relative to atmospheric pressure. Atmospheric pressure was determined with a mercury barometer. 
Twelve copper-constantan thermocouples were used in these measurements. The main thermocouples of interest were placed in the midale of the glass flow tube $6.3 \mathrm{~cm}$ upstream and downstream of the orifice, respectively. Other thermocouples were placed throughout the apparatus to make sure that the interior of the thermostated box had reached thermal equilibrium.

Procedure. The gas and gas-solid Joule-Thomson data were taken in a temperature range of $298-303 \mathrm{~K}$. The initial procedure which was used for a solid aerosol was the same as for a pure gas. After the gas flowed for about 15 minutes and the data were obtained for the pure gas, the aerosol generator was started. While a gas-powder mixture was flowing the measurements of $\Delta T, \Delta P, T$, and $P$ were made. In addition, the average concentration of the aerosol was calculated from the mass of powder transported to the collector and moles of gas flowed through the flowmeter during the time interval the aerosol generator was operating.

Results. Experimental Joule-Thomson data were obtalned for 12 gas-solid systems (ref. 9). The gaseous component was elther argon, nitrogen, or carbon dioxide. The particulate component was either Mexican Graph1te (MG), Nuchar S-C (SC), Nuchar S-A (SA), or Super Sorb (SS). Two sets of data were taken for each gas-solid system including values of $\Delta T, \Delta P, P$, and $T$. One set of data was obtained from measurements made when the aerosol generator was not operating and thus represents pure gas flow. The powders were selected to span a range of surface area and porosity. The nitrogen BET surface areas were 26, 903, 1661, and $3169 \mathrm{~m}^{2} / \mathrm{g}$ for Mexican Graph1te (MG), Nuchar S-C (SC), Nuchar S-A (SA), and Super Sorb (SS), respect1vely. Pore size distributions were determined. The pore volumes for pores in the 2 to $60 \mathrm{~nm}$ range were $0.118,0.572,0.925$, and $0.797 \mathrm{~cm}^{3} / \mathrm{g}$ for $M G, S C, S A$, and SS, respectively. The results of the measurements are given in Table I which includes values for ${ }_{\mathrm{JT}}$ determined at $302 \mathrm{~K}$ and a loading (w) of 10 grams of solid per mole of gas. Gas-solid chromatography was used to determine values of $B_{2 s}$ between 265 and $325 \mathrm{~K}$ for each of these systems. From gas-solid chromatography measurements (ref. 10) the temperature dependences of the second gas-solid virial coefficients were determined. As expected, 1t was found that in $B_{2 s}$ versus $1 / T$ was linear.

\section{DISCUSSION}

The effectiveness of the powders in producing a gas-solid Joule-Thomson cooling effect are in the order SS $>S A>S C>M G$. The effectiveness of the gases in producing a gas-sol1d Joule-Thomson cooling effect was in the order $\mathrm{CO}_{2}>\mathrm{N}_{2}>\mathrm{Ar}$. Basically, these results correlate with the surface area of the powders and the expected strength of the gas-solid interaction. A direct comparison of the theoretical predictions and the experimental results found for the gas-solid Joule-Thomson effect requires an independent measurement of the second gas-solid virial coefficient and its temperature dependence for each of the twelve gas-solid systems which had been studied. Using the results from the gas-solid chromatography values were predicted and are included in Table $I$. A comparison of the values obtained from the gas-solid Joule-Thomson experiments (meas) and the values obtained from the gas-solid chromatography (pred) experiments reveals that the former values are always less than the latter values.

The apparent discrepancies between these two methods may be reconciled by considering the influence of the gas-solid interaction energy on the kinetics of desorption. The Joule-Thomson coefficient, as defined, is an equilibrium thermodynamic property. In a Joule-Thomson experiment for a pure gas, the flowing gas reaches equilibrium just beyond the orifice. Because the molecules are weakly interacting through the van der Waals forces, there is no signiflcant barrier to prevent the rapid attalnment of equilibrium. However, the gas-solid interaction energies are 10 to 20 times as strong as the pure gas interaction energies. For the gas-powder systems, especially the porous powders, there is an energetic barrier to the desorption process. Thus as the gas-solid aerosol flows past the downstream themocouple, the equilibrium distribution of adsorbed and desorbed gas molecules may not have been attained. Therefore, the observed deltaT is smaller than the expected value based on equilibrium measurements such as those made with gas-solid chromatography. 
Based on an Arrhenius type relation, the rate of desorption should be dependent on the exponential of the energy of activation of desorption. If the attenuation of the expected (GSC) Joule-Thomson coefficient is due to the time required for desorption in the actual gas-solid Joule-Thomson experiment, then the ratio of $\mu_{\mathrm{gs}}$ (meas)/ $\mu_{\mathrm{gs}}$ (pred) should be related to the rate of desorption. The energy of activation for desorption can be approximated by the isosteric heat since the gas-solid systems are in the region of low coverage. Therefore, it is expected that the logarithms of $\mu_{\mathrm{gs}}$ (meas)/ $\mu_{\mathrm{gs}}$ (pred) will be linearly proportional to the isosterlc heats of gas-porous carbon systems. Figure 3 shows the suggested linear relation. An additional consideration is that higher order virial coefficlents may be required to correctly predict values of "gs's even at
these temperatures and coverages.

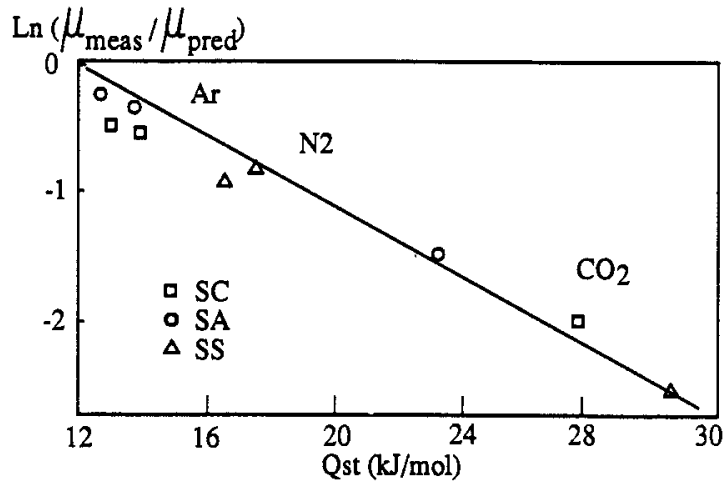

Fig. 3. Ratio of measured to predicted gas-solid Joule-Thomson coefficients as related to the isosteric heat of adsorotion.
Table I. Joule-Thomson Coefficients at $302 \mathrm{~K}$ and 10 grams of solid per mole of gas. (units are deg/atm)

\begin{tabular}{|c|c|c|}
\hline Gas-5olid & Heasurad & Predicted \\
\hline$A I-\mathbb{L G}$ & -0.05 & $0.2 B$ \\
\hline$-\mathbf{S A}$ & 0.88 & 1.01 \\
\hline$-5 s$ & 1.15 & 1.65 \\
\hline YZ-HG & 0.03 & 0.18 \\
\hline$-5 A$ & 0.64 & 0.81 \\
\hline-55 & 0.93 & 1.42 \\
\hline COZ-HG & 0.84 & 0.96 \\
\hline$-5 A$ & 3.27 & $1 \mathrm{~B} \cdot \mathrm{B}$ \\
\hline-55 & 5.14 & 19.8 \\
\hline
\end{tabular}

\section{CONCLUSION}

It is clear from the preliminary theoretical and experimental results presented that the gas-solid Joule-Thomson effect is one worthy of further exploration. The effect appears to be sufficiently large that measurements of gas-solid Joule-Thoms on coefficients could provide information about the nature of the interaction potential between gases and solids in addition to possibly having a practical value in thermodynamic cycles for heat engines and other thermodynamic devices (ref. 11). There can be no doubt that gassolid aerosols can be viewed as flulds having very interesting and unique thermal properties. The discrepancies between the predicted and measured values and the clear trend associated with those discrepancles are areas for further study and investigation.

\section{REFERENCES}

1. R. A. Plerotti and T. R. Rybolt, J. Chem. Phys., 80, 3826 (1984).

2. T. L. H1ll, Statistical Mechanics, McGraw-H111, New York (1956).

3. W. A. Steele and G. D. Halsey, Jr., J. Chem. Phys., 22, 979 (1954); W. A. Steele, The Gas-Sol1d Interface, ed. E. A. Fiood, Vol. I, pp. 199-220, Dekker, New York, (1967).

4. R. A. Pierotti and H. E. Thomas, Surface and Colloid science ed. E. Mat1jevic, Vol. IV, pp. 97-259, Wllley-Intersclence, New York, (1971).

5. J. H. Cole, D. H. Everett, C. T. Marshall, A. R. Panlego, J. C. Powl and F. R. Reinoso, J. Chem. Soc. Faraday Trans., 170, 2154 (1984).

6. C. C. Yang, Ph.D. Thesis, Georgia Institute of Technology (1979).

7. J. R. Sams, G. Constabaris, G. D. Halsey, Jr., J. Phys. Chem., 64, $1689(1960)$.

8. T. R. Rybolt, Ph.D Thesis, Georgia Institute of Technology (1981).

9. T. R. Rybolt and R. A. Plerott1, A.I.Ch.E.Journal, 30, 510 (1984).

10. T. R. Rybolt and R. A. Plerotti, J. Phys. Chem., 88, 2398 (1984).

11. R. A. P1erott1 and T. R. Rybolt, U.S. Patent 4,321,799 (1982). 